\title{
ON SUBINJECTIVITY DOMAINS OF PURE-INJECTIVE MODULES
}

\author{
YILMAZ DURĞUN
}

\begin{abstract}
As an alternative perspective on the injectivity of a pure-injective module, a pure-injective module $M$ is said to be pi-indigent if its subinjectivity domain is smallest possible, namely, consisting of exactly the absolutely pure modules. A module $M$ is called subinjective relative to a module $N$ if for every extension $K$ of $N$, every homomorphism $N \rightarrow M$ can be extended to a homomorphism $K \rightarrow M$. The subinjectivity domain of the module $M$ is defined to be the class of modules $N$ such that $M$ is $N$-subinjective. Basic properties of the subinjectivity domains of pure-injective modules and of pi-indigent modules are studied. The structure of a ring over which every pure-injective (resp. simple, uniform, indecomposable) module is injective or subinjective relative only to the smallest possible family of modules is investigated. This work is a natural continuation to recent papers that have embraced the systematic study of the subinjective and subprojective domains of modules.
\end{abstract}

\section{INTRODUCTION}

Throughout, $R$ will denote an associative ring with identity. As usual, we denote by $M o d-R$ the category of right $R$-modules. Some recent work in module theory has focused on classical injectivity in order to consider the extent of injectivity of modules from a fresh perspective. Recall that a module $M$ is said to be $N$ subinjective if for every extension $K$ of $N$ and every homomorphism $f: N \rightarrow M$ there exists a homomorphism $h: K \rightarrow M$ such that $\left.h\right|_{N}=f$. For a module $M$, the subinjectivity domain of $M, \underline{\mathfrak{I n}}^{-1}(M)$, is defined to be the collection of all modules $N$ such that $M$ is $N$-subinjective, that is $\mathfrak{I n n}^{-1}(M)=\{N \in \operatorname{Mod}-R \mid$ $\mathrm{M}$ is $\mathrm{N}$-subinjective (see [4). It is clear that a module $M$ is injective if and only if $\underline{\mathfrak{n}}^{-1}(M)=\operatorname{Mod}-R$. If $N$ is injective, then $M$ is $N$-subinjective. So, the smallest possible subinjectivity domain is the class of injective modules. While traditionally the study of non-injective modules has emphasized those modules that are as injective as possible, the recent has been made to understand also the diametrical opposite notion of modules which are subinjective only with respect to the smallest possible class of modules, i.e. the class of injective modules. One of the notions thus introduced is that of an indigent module (see 4 4). Presently, it is not known whether indigent module exists for an arbitrary ring, but an affirmative answer is known for some rings, such as Noetherian rings [9. In [1, the authors studied ring $R$ with the property that every non-injective right module is indigent. Indigent modules have been recently studied in 2]. In [14, inspired by the notion

Date: April 3, 2019.

2010 Mathematics Subject Classification. 18G25; 16D50; 16D70.

Key words and phrases. pure-injective module; subinjective domain; pi-indigent module; absolutely pure module. 
of relative subinjectivity, pure-subinjectivity domain of a module is introduced and studied. A module $M$ is said to be $N$-pure-subinjective if for every pure extension $K$ of $N$ and every homomorphism $f: N \rightarrow M$ there exists a homomorphism $h: K \rightarrow M$ such that $\left.h\right|_{N}=f$. The pure-subinjectivity domain of $M$ consists of those modules $N$ such that $M$ is $N$-pure-subinjective.

In this paper, motivated by these above-mentioned papers, we address some questions on pure-injective modules. The first question discussed here is: What is the structure of a ring $R$ with the property that every non-injective pure injective left module is subinjective relative only to the smallest possible class of modules? To approach this problem, we use the following observation: The smallest possible subinjectivity domain of a pure injective module is the class of absolutely pure (fpinjective) modules. To keep in line with [4, we refer to these pure injective modules as pure injectively indigent, or pi-indigent for short. In contrast to indigent modules, such pure injective modules exist over any ring (Proposition 4). We prove that if such a ring is two-sided coherent then it is either two-sided semihereditary or right IF-ring (Proposition 12). We also prove that if such a ring is left noetherian left nonsingular then every non-flat left $R$-module is i-test and $R$ is left n-saturated ring (Lemma 16).

Second, we study the structure of a ring $R$ with the property that every noninjective pure injective right module is indigent. We prove that if such a ring is right noetherian then it is isomorphic to the direct product of a semisimple Artinian ring and an indecomposable ring $A$ such that (i) $A$ is right n-saturated matrix ring over local $Q F$-ring; or, (ii) $A$ is hereditary Artinian serial ring with $J(A)^{2}=0$; or, (iii) $A$ is $S I$-ring with $\operatorname{Soc}\left({ }_{A} A\right)=0$ (Theorem 17).

Finally, we study the structure of a ring $R$ with the property that every noninjective simple right module is indigent. In [1, Alizade, Büyükaşik and Er also investigate when non injective simple modules are indigents. We improve their results proving that every non-injective simple right $R$-module is indigent if and only if (i) $R$ is a right V-ring; or, (ii) $R$ is right Hereditary righ Noetherian ring and, for any right $R$-module $M$, either $M$ is indigent or $\operatorname{Soc}(M)=\operatorname{Soc}(N)$, where $N$ is the largest injective submodule of $M$; or, (iii) $R \cong S \times T$, where $S$ is semisimple Artinian ring and $T$ is an indecomposable matrix ring over a local QF-ring (Theorem 18). We show that, for a right nonsingular ring which is not right V-ring, every noninjective simple right $R$-module is indigent if and only if every non-injective uniform (or indecomposable) right $R$-module with nonzero socle is indigent (Proposition 201). Moreover, we show that, for commutative nonsingular ring which is not Vring, every non-injective simple module is indigent if and only if every non-injective singular module is indigent (Proposition 22).

For a module $M, E(M), \operatorname{Soc}(M)$, and $Z(M)$ will stand for the injective hull, the socle and the singular submodule respectively. For any ring in our discussion, $J$ will stand for the Jacobson radical of that ring. For a module $M$, the character module $\operatorname{Hom}_{\mathbb{Z}}(M, \mathbb{Q} / \mathbb{Z})$ is denoted by $M^{+}$. For other concepts and problems not mentioned here, For other concepts or background materials, we refer the reader to [10, 19.

\section{The SubinjeCtivity domain of A PURE-InJeCtive Module}

The notion of purity has an significant role in module theory and model theory since it was presented in the literature (see [7,24]). There are several generalizations 
of the notion of purity(see [18,20,24]). For a survey on purities, we refer the reader to 21 .

Let $\delta$ be a class of right $R$-modules. An exact sequence $0 \rightarrow A \rightarrow B \rightarrow C \rightarrow 0$ a sequence of left $R$-modules is called $\delta$-pure, if every member of $\delta$ is flat with respect to this sequence; i.e., the induced homomorphism $M \otimes B \rightarrow M \otimes C$ is a monomorphism for each $M \in \delta$. A submodule $A$ of an $R$-module $B$ is called a $\delta$-pure submodule if the exact sequence $0 \rightarrow A \rightarrow B \rightarrow C \rightarrow 0$ is $\delta$-pure. An $R$-module $M$ is said to be $\delta$-pure injective (resp. projective) if $M$ has the injective (resp. projective) property relative to each $\delta$-pure exact sequence. An $R$-module $N$ is said to be absolutely $\delta$-pure (resp. $\delta$-flat) if every exact sequence starting (resp. ending) with $N$ is $\delta$-pure. If $\delta=\{M\}$, we say $M$-pure instead of $\delta$-pure.

If we take for $\delta$ the class of all (or even only finitely presented) right $R$-modules, then we get the classical purity, which is usualy called the Cohns purity. In this case, $\delta$-pure exact, $\delta$-pure injective, $\delta$-pure projective, absolutely $\delta$-pure and $\delta$-flat are commonly called pure exact, pure injective, pure projective, absolutely pure (or fp-injective) and flat, respectively.

The author in [4] show for a module $M$ to be $N$-subinjective, one only needs to extend maps to $E(N)$. We have improved this result as follows.

Lemma 1. Let $M, N \in M o d-R$. Then the following conditions are equivalent.

(1) $M$ is $N$-subinjective.

(2) For every $f: N \rightarrow M$ and every monomorphism $g: N \rightarrow F$ with $F \in$ $\mathfrak{I n}^{-1}(M)$, there exists $h: F \rightarrow M$ such that $h g=f$.

(3) There is a monomorphism $g: N \rightarrow F$ with $F \in \underline{\mathfrak{I n}}^{-1}(M)$, such that for every $f: N \rightarrow M$, there exists $h: F \rightarrow M$ such that $h g=f$.

Proof. (1) $\Rightarrow(2)$ and (2) $\Rightarrow$ (3) follows by [4, Lemma 2.2]. (3) $\Rightarrow(1)$ Let $f: N \rightarrow$ $M$ be a homomorphism. By assumption, there is a monomorphism $g: N \rightarrow F$ with $F \in \underline{\mathfrak{I n}}^{-1}(M)$ and a homomorphism $h: F \rightarrow M$ such that $h g=f$.

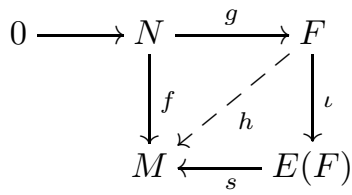

Since $M$ is $F$-subinjective, there exists a homomorphism $s: E(F) \rightarrow M$ such that $s \iota=h$. It is clear that $\iota g$ is a monomorphism and $f=s \iota g$. Then $M$ is $N$-subinjective by [4, Lemma 2.2].

The character module $N^{+}$of a right $R$-module $N$ is a pure injective left $R$ module [10, Proposition 5.3.7]. It is known that $(M \otimes N)^{+} \cong \operatorname{Hom}\left(N, M^{+}\right)$by the adjoint isomorphism, (see [19]). Then, the following obsevation is clear.

Proposition 2. Let $M$ be a right $R$-module and $N$ a left $R$-module. Then, $N$ is absolutely $M$-pure if and only if $M^{+}$is $N$-subinjective.

Note that a pure injective module $M$ is injective if and only if $\mathfrak{I n n}^{-1}(M)=$ $\operatorname{Mod}-R$. If $N$ is absolutely pure, then $M$ is vacuously $N$-subinjective. So, the smallest possible subinjectivity domain of a pure injective module is the class of absolutely pure modules. 
Proposition 3. Let PI be a class of all pure injective left $R$-modules. Then, $\bigcap_{M \in P I} \underline{\mathfrak{I n}}^{-1}(M)=\{N \in R-M o d \mid N$ is absolutely pure $\}$

Proof. Let $K \in \bigcap_{M \in P I} \underline{\mathfrak{I n}}^{-1}(M)$. In particular, for each finitely presented right $R$ module $M, M^{+}$is $K$-subinjective. By the preceding proposition, $K$ is absolutely $M$ pure for each finitely presented right $R$-module $M$, and hence $K$ is absolutely pure module. The converse folows from the discussion in the preceding paragraph.

As subinjectivity domains of pure-injective modules clearly include all absolutely pure modules, a reasonable opposite to injectivity of pure-injective modules in this context is obtained by considering pure-injective modules whose subinjectivity domain consists of only absolutely pure modules.

Definition 1. We will call a pure-injective module $M$ pure injectively indigent (or a pi-indigent) module in case $\mathfrak{\mathfrak { n }}^{-1}(M)=\{A \in \operatorname{Mod}-R \mid A$ is absolutely pure $\}$.

Certainly, the first problem that comes to mind with the introduction of the notion of pi-indigent modules is whether such pure injective modules exist over all rings. For the remainder of this paper, let $\mathfrak{P I}:=\prod_{S_{i} \in \Gamma} S_{i}^{+}$, where $\Gamma$ be a complete set of representatives of finitely presented left $R$-modules.

Proposition 4. $\mathfrak{P I}$ is a pi-indigent right $R$-module.

Proof. A right $R$-module $N$ is absolutely pure if and only if $N \otimes M \rightarrow E(N) \otimes M$ is a monomorphism for each finitely presented left $R$-module $M$, i.e. $N$ is absolutely $M$-pure for each finitely presented left $R$-module $M$ (see, [10, 6.2.3]). Then, $\mathfrak{P I}$ is a pi-indigent right $R$-module by Proposition 2 and Lemma 1

Note that a ring $R$ is right Noetherian if and only if all absolutely pure right $R$-modules are injective (see [17, Theorem 3]). We have the following observation by Proposition 4 .

Corollary 5. Let $R$ be a ring. $R$ is right Noetherian if and only if $\mathfrak{P I}$ is an indigent module.

A ring $R$ is right semihereditary if and only if every homomorphic image of an absolutely pure right $R$-module is absolutely pure (see [17, Theorem 2]). In general, the subinjectivity domain of a pure-injective module is not closed with respect to homomorphic images. Consider for example the right $R$-module $\mathfrak{P I}$ over a ring $R$ which is not right semihereditary. Since $R$ is not right semihereditary ring, the subinjectivity domain of $\mathfrak{P I}$ is not closed with respect to homomorphic images by Proposition 4.

Proposition 6. A ring $R$ is right semihereditary if and only if the subinjectivity domain of any pure injective right $R$-module is closed under homomorphic images.

Proof. Assume that $R$ is a right semihereditary ring. Suppose a pure injective right module $M$ is $N$-subinjective for a right module $N$. Let $K$ be a submodule of $N$ 
and $f: N / K \rightarrow M$ a homomorphism. Consider the following diagram:

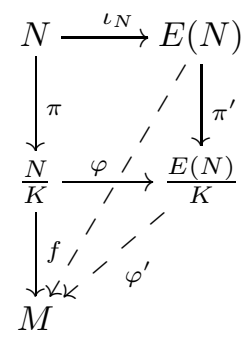

where $\pi, \pi^{\prime}$ are canonical epimorhisms. Since $M$ is $N$-subinjective, there is a $\varphi$ : $E(N) \rightarrow M$ such that $\varphi_{\left.\right|_{N}}=f \pi$. Clearly $f \pi(K)=0$, and so $K \subseteq \operatorname{Ker}(\varphi)$. Then, by factor theorem, there exists a homomorphism $\varphi^{\prime}: E(N) / K \rightarrow M$ such that $\varphi^{\prime} \pi^{\prime}=\varphi$. Clearly, $\varphi_{\left.\right|_{N / K} ^{\prime}}^{\prime}=f$ and, by semihereditary of $R, \frac{E(N)}{K}$ is absolutely pure. Now, $M$ is $\frac{N}{K}$-subinjective by Lemma 1. This proves the necessity.

Conversely suppose the subinjectivity domain of any pure injective right module is closed under homomorphic images. In particular, the subinjectivity domain of the module $\mathfrak{P I}$ is also closed under homomorphic image. But $\mathfrak{P I}$ is pi-indigent, and hence absolutely pure modules are closed under homomorphic image, this implies, by [17, Theorem 2], that $R$ is a right semihereditary ring.

A ring $\mathrm{R}$ is said to be a von Neumann regular ring if for each $a \in R$ there is an $r \in R$ such that $a=$ ara. Every right (left) $R$-module is absolutely pure if and only if $R$ is a von Neumann regular ring. The proof of the following is obvious from the definitions.

Proposition 7. The following statements are equivalent for a ring $R$ :

(1) $R$ is von Neumann regular.

(2) Every (non-zero) pure-injective right (left) $R$-module is pi-indigent.

(3) There exists an injective pi-indigent right (left) $R$-module.

In the rest of this article, unless otherwise stated, all rings will be non von Neumann regular.

Proposition 8. Let $I$ be a non flat right ideal of a ring $R$. If $I^{+}$is pi-indigent, then $R$ is a left absolutely pure.

Proof. Recall that a right $R$-module $M$ is flat if and only if $M^{+}$is injective ([19, Theorem 3.52]). Therefore, $I^{+}$is not injective. Assume that $I^{+}$is pi-indigent. Note that $I^{+}$is an epimorphic image of the injective module $R^{+}$. But since $I^{+}$is pi-indigent, $R$ must be left absolutely pure by [ 8 , Lemma 2.3$]$.

The weak global dimension of $R, w D(R)$, is less than or equal 1 if and only if every submodule of a flat right (left) $R$-module is flat if and only if every (finitely generated) right (left) ideal is flat, (see [19, 9.24]).

Corollary 9. If $I^{+}$is injective or pi-indigent for every finitely generated right ideal $I$ of $R$, then $R$ is a left absolutely pure or $w D(R) \leq 1$. 


\section{EVERY PURE-INJECTIVE MODULE IS INJECTIVE OR PI-INDIGENT}

This section deals with the structure of a ring $R$ with the property that every non-injective pure-injective module is subinjective relative only to absolutely pure modules. Note that a pure-injective right module is injective if it is also absolutely pure. The following proposition states an obvious fact without proof. We will use this proposition freely in the sequel.

Proposition 10. The following conditions are equivalent for a ring $R$ :

(1) Every pure-injective left module is injective or pi-indigent;

(2) Every pure-injective left module is absolutely pure or pi-indigent.

For easy reference, $(\mathrm{P})$ stands for the property that $R$ satisfies the equivalent conditions of Proposition 10 .

The following Proposition can be proved using standard arguments. We omit its proof, which has much in common with the proof of [10, 6.2.3].

Proposition 11. The following statements are equivalent for any given modules $M_{R}$ and ${ }_{R} N$.

(1) ${ }_{R} N$ is absolutely $M_{R}$-pure.

(2) $M \otimes N \rightarrow M \otimes E(N)$ is a monomorphism.

(3) There exists an absolutely pure extension $E$ of $N$ such that $M \otimes N \rightarrow M \otimes E$ is a monomorphism.

Proposition 12. Let $R$ be a two-sided coherent ring satisfying the condition $(P)$. Then $R$ is either $R$ is two-sided semihereditary or $R$ is right IF-ring.

Proof. By Corollary 9] $R$ is $w D(R) \leq 1$ or a left absolutely pure. In the former case, by the coherence of $R, R$ is a two-sided semihereditary ring. In the latter case, by [22, Proposition 4.2], $R$ is right IF-ring, i.e. every absolutely pure right module is flat.

In [12, Holston et al. are interested in the projective analog of the notion of subinjectivity. Namely, a module $M$ is said to be $N$-subprojective if for every epimorphism $g: B \rightarrow N$ and homomorphism $f: M \rightarrow N$, then there exists a homomorphism $h: M \rightarrow B$ such that $g h=f$. For a module $M$, the subprojectivity domain of $M, \mathfrak{P r}^{-1}(M)$, is defined to be the collection of all modules $N$ such that $M$ is $N$-subprojective, that is $\mathfrak{P \mathfrak { r }}^{-1}(M)=\{N \in \operatorname{Mod}-R \mid \mathrm{M}$ is N-subprojective $\}$.

Remark 13. Let $M_{R}$ be a finitely presented module, that is, $M$ has a free presentation $F_{1} \stackrel{f}{\rightarrow} F_{0} \rightarrow M \rightarrow 0$ where $F_{0}$ and $F_{1}$ are finitely generated free modules. If we apply the functor $\operatorname{Hom}_{R}(., R)$ to this presentation, we obtain the sequence

$$
0 \rightarrow M^{*} \rightarrow F_{0}^{*} \rightarrow F_{1}^{*} \rightarrow \operatorname{Tr}(M) \rightarrow 0
$$

where $\operatorname{Tr}(M)$ is the cokernel of the dual map $F_{0}^{*} \rightarrow F_{1}^{*}$. Note that, $\operatorname{Tr}(M)$ is a finitely presented left $R$-module. The left $R$-module $\operatorname{Tr}(M)$ is called the AuslanderBridger transpose of the right $R$-module $M$, (see [3]).

Proposition 14. 9. Proposition 2.7] Let $M_{R}$ be a finitely presented module. The following properties hold for any modules $N_{R}$ and ${ }_{R} K$ :

(1) $K$ is absolutely $M$-pure if and only if $\operatorname{Ext}(\operatorname{Tr}(M), K)=0$.

(2) $M$ is $N$-subprojective if and only if $\operatorname{Tor}(N, \operatorname{Tr}(M))=0$. 
Corollary 15. Let $M_{R}$ be a finitely presented module. The following properties hold for any modules $N_{R}$ and ${ }_{R} K$ :

(1) $K \in \underline{\mathfrak{I n}}^{-1}\left(M^{+}\right)$if and only if $K^{+} \in \mathfrak{P r}^{-1}(M)$.

(2) $N \in \underline{\mathfrak{P r}}^{-1}(M)$ if and only if $N^{+} \in \underline{\mathfrak{I n}}^{-1}\left(M^{+}\right)$.

Proof. (1) Let $K \in \mathfrak{I n}^{-1}\left(M^{+}\right)$. Then $K$ is absolutely $M$-pure by Proposition 11] and so, by Proposition 14, $\operatorname{Ext}(\operatorname{Tr}(M), K)=0$. Since $\operatorname{Tor}\left(K^{+}, \operatorname{Tr}(M)\right) \cong$ $\operatorname{Ext}(\operatorname{Tr}(M), K)^{+}$by [19, Theorem 9.51], Tor $\left(K^{+}, \operatorname{Tr}(M)\right)=0$. Hence, $K^{+} \in$ $\mathfrak{P r}^{-1}(M)$ by Proposition 14, and vice versa.

The proof of (2) is similar to the proof of (1).

A module $M$ is called a Whitehead test module for injectivity (i-test module) if $N$ is injective whenever $\operatorname{Ext}_{R}^{1}(M, N)=0$ (23]). A non-semisimple $\operatorname{ring} R$ is said to be fully(resp. n-) saturated provided that all non-projective (resp. finitely generated) modules are i-test. In [1, Theorem 16], authors proved that a non von Neumann regular ring $R$ is right fully saturated if and only if all non-injective modules are indigent.

Lemma 16. Let $R$ be a left noetherian left nonsingular ring which is satisfying the condition $(P)$. Then, every non-flat left $R$-module is i-test. In particular, $R$ is left $n$-saturated ring.

Proof. Recall that a module is flat if and only if its character module is injective. Hence, the subprojectivity domain of any finitely presented right module under the assumption $(\mathrm{P})$, consists precisely of the flat modules by Corollary $15(2)$.

By Corollary 9, $R$ is left fp-injective or $w D(R) \leq 1$. In the former case, $R$ is QFring by [11, Theorem 2.2], yielding a contradiction because $R$ is left nonsingular. In the latter case, by the noethernity of $R, R$ is left hereditary. Let $M$ be any non flat left $R$-module. $M$ has a finitely presented submodule which is not projective by [19, Corollary 3.49], say $S$. Assume that $\operatorname{Ext}(S, N)=0$ for some left $R$-module $N$. Note that $\operatorname{Tr}(\operatorname{Tr}(S)) \cong S$. By Proposition 14 and the adjoint isomorphism, $\operatorname{Tr}(S)^{+}$ is $N$-subinjective. But, by the property $(\mathrm{P}), \operatorname{Tr}(S)^{+}$is pi-indigent, and hence $N$ is injective. Therefore, $S$ is i-test, and hence $M$ is also i-test by [9, Proposition 4.3]. Furthermore, by the noethernity of $R, R$ is left n-saturated ring.

The projective analog of indigent modules was considered in [12, namely, pindigent modules. A module $M$ is p-indigent if $\mathfrak{P r}^{-1}(M)$ consists precisely of the projective modules.

Theorem 17. Let $R$ be a right Noetherian ring. Assume that every non-injective pure-injective left $R$-module is indigent. Then $R \cong S \times T$, where $S$ is a semisimple artinian ring and $T$ is an indecomposable ring satisfying one of the following conditions:

(1) $T$ is right n-saturated matrix ring over local $Q F$-ring; or,

(2) $T$ is hereditary Artinian serial ring with $J(T)^{2}=0$; or,

(3) $T$ is SI-ring with $\operatorname{Soc}\left({ }_{T} T\right)=0$.

Proof. If every pure-injective left $R$-module is injective, then $R$ is von Neumann regular. Assume that there exists at least one non-injective pure injective indigent left $R$-module. Since each pure-injective left $R$-module is $N$-subinjective for every 
absolutely pure left module $N$, all absolutely left $R$-modules are injective, yielding that $R$ is a left noetherian ring by [17, Theorem 3].

By Corollary 9, $R$ is left absolutely pure or left semihereditary ring. In the former case $R$ is $Q F$-ring by [11, Theorem 2.2]. Let $M$ be a non-projective finitely generated right $R$-module. For a right $R$-module $N$, by Corollary 15, $N \in \mathfrak{P r}^{-1}(M)$ if and only if $N^{+} \in \mathfrak{I n}^{-1}\left(M^{+}\right)$. By our assumption, $M^{+}$is indigent, and thus $N^{+}$ is injective. By [6, Theorem 4], $N$ is projective. Then, every finitely generated right R-module is projective or p-indigent. This implies, by [9, Theorem 4.1], that there is a ring direct sum $R \cong S \times T$, where $S$ is semisimple Artinian ring and $T$ is an indecomposable ring which is right n-saturated matrix ring over a local QF-ring.

In the latter case, $R$ is left hereditary ring by the noetherianity. $R$ is not right $I F$-ring, because otherwise so would be $R$ is left fp-injective by [11, Corollary 8.2]. Then, by noethernity of $R, R$ is both $Q F$ and hereditary, and this implying $R$ is semisimple artinian, a contradiction. Therefore, $R$ has a non-flat injective right $R$-module, say $E$. The character module $E^{+}$is a nonsingular left module by [6. Theorem 2] and [9, Proposition 4.4]. Note that $E^{+}$is not injective, because otherwise so would be $E$ is flat by [6. Theorem 2], a contradiction. Then, $E^{+}$is indigent nonsingular module by our assumption, and thus $R$ is a left $S I$-ring, i.e. every singular left module is injective.

Now, we will show that $R$ has a unique singular simple right $R$-module up to isomorphism. Let $A$ be a non-projective simple right $R$-module. By noethernity of $R$, it is finitely presented. For a right $R$-module $N$, by Corollary 15, $N \in$ $\mathfrak{P r}^{-1}(A)$ if and only if $N^{+} \in \mathfrak{I n}^{-1}\left(A^{+}\right)$. Note that $A^{+}$is not injective, otherwise, by [19, Theorem 3.52], $A$ is flat. But $A$ is finitely presented, so $A$ becomes projective, a contradiction. Let $B$ be a singular simple right $R$-modules. Assume that $A$ and $B$ are not isomorphic. Then, $A$ is clearly $B$-subprojective, and so $B$ is flat. Since $R$ is right Noetherian, $B$ is finitely presented, and so it is projective by [19, Corollary 3.58], contradicting the singularity of $B$. Thus, $R$ has a unique singular simple right module $A$ up to isomorphism.

We claim that every finitely generated left $R$-module is a direct sum of a projective module and an injective module, i.e., $R$ is a left FGPI ring (see [13]). Let $M$ be a finitely generated left $R$-module. Recall that $R$ is left Noetherian ring, and so $M$ is finitely presented. Consider the exact sequence $0 \rightarrow Z(M) \rightarrow M \rightarrow M / Z(M) \rightarrow 0$. Since $R$ is left $S I$-ring, $M \cong Z(M) \oplus(M / Z(M))$. $M / Z(M)$ is a nonsingular finitely presented module. Since $R$ is left hereditary left noetherian ring, it is flat by [9, Proposition 4.4], and so projective. Therefore, $R$ is a left FGPI ring By [13. Theorem 8], $R \cong U \times V$ where $U$ is left Artinian left $S I, V$ is left and right $S I$-ring with $\operatorname{Soc}\left({ }_{V} V\right)=0$. If $A$ is right $U$-module, then $V$ must be zero, and hence $R \cong U$ is left Artinian. Then, $R$ is Artinian by the right noetherianity. By [9. Theorem 4.1], $R \cong S \times T$, where $S$ is a semisimple artinian ring and $T$ satisfy (2) in Theorem [17. In case $S$ is $V$-module, $U$ is semisimple and $V$ satisfy (3) in Theorem 17.

\section{Ring Whose Simple modules ARE INDigent oR INJECTIVE}

In [1, the authors interested with the structure of rings over which every noninjective module is indigent. In this section, we deal with the structure of rings over which every non-injective simple (respectively, singular, uniform, indecomposable) 
module is indigent. Recall that a ring $R$ is called right $V$-ring if all simple right R-modules are injective.

Theorem 18. Let $R$ be a ring. The following are equivalent.

(1) Every non-injective simple right $R$-module is indigent.

(2) One of the following statements hold:

(i) $R$ is a right V-ring; or,

(ii) $R$ is right Hereditary righ Noetherian ring and, for any right $R$-module $M$, either $M$ is indigent or $\operatorname{Soc}(M)=\operatorname{Soc}(N)$, where $N$ is the largest injective submodule of $M$; or,

(iii) $R \cong S \times T$, where $S$ is semisimple Artinian ring and $T$ is an indecomposable matrix ring over a local QF-ring.

Proof. (1) $\Rightarrow(2)$ Suppose every non-injective simple right $R$-module is indigent. If all simple modules are injective, then $R$ is a right $V$-ring. Now suppose, there is a non-injective simple right $R$-module $U$. Then $U$ is indigent by the hypothesis. If $U^{\prime}$ is any simple module which is not isomorphic to $U$, then $\operatorname{Hom}\left(U^{\prime}, U\right)=0$. That is, $U$ is $U^{\prime}$-subinjective, and so $U^{\prime}$ must be injective because $U$ is indigent. Thus the ring has a unique non-injective simple module up to isomorphism, say $U$. Through injective modules, we have the following two cases:

Case I. $\operatorname{Hom}(E, U)=0$ for each injective right $R$-module $E$. Then, $U$ is $N$ subinjective for any right $R$-module $N$ if and only if $\operatorname{Hom}(N, U)=0$. Let $N$ be a injective right $R$-module and let $K$ a submodule of $N$. $\operatorname{Hom}(N / K, U)=0$, since $0 \rightarrow \operatorname{Hom}(N / K, U) \rightarrow \operatorname{Hom}(N, U)=0$. Then $N / K$ is injective by indiginity of $U$. This implying $R$ is right Hereditary. Let $\left\{E_{i}\right\}_{i \in I}$ be an arbitrary family of injective modules. By isomorphism $\operatorname{Hom}\left(\oplus_{i \in I} E_{i}, U\right) \cong \prod_{i \in I} \operatorname{Hom}\left(E_{i}, U\right)=0, U$ is $\oplus_{i \in I} E_{i}$-subinjective. As $U$ is indigent, $\oplus_{i \in I} E_{i}$ must be injective. So that the $\operatorname{ring} R$ is Noetherian.

Recall that a right module is called reduced if it has no nonzero injective submodule. Let $M$ be a right $R$-module. Since $R$ is right Hereditary and right Noetherian, $M=N \oplus M^{\prime}$ for some $M^{\prime}, N \leq M$, where $M^{\prime}$ is the reduced part of $M$ and $N$ is the largest injective submodule of $M$ (see [15]). Note that, as $R$ is hereditary, $\operatorname{Hom}\left(U^{\prime}, M^{\prime}\right)=0$ for every injective simple right $R$-module. There are two cases for $M^{\prime}$ by $U$ : either $\operatorname{Hom}\left(U, M^{\prime}\right)=0$ or $\operatorname{Hom}\left(U, M^{\prime}\right) \neq 0$. In the former case, $\operatorname{Soc}\left(M^{\prime}\right)=0$, and hence $\operatorname{Soc}(M)=\operatorname{Soc}(N)$. For the latter case, assume that $M^{\prime}$ is not indigent. Then there is a non injective right $R$-module $K$ such that $M^{\prime}$ is $K$-subinjective. $\operatorname{Hom}(K, U) \neq 0$, otherwise $K$ is injective by indiginity of $U$. Then $M^{\prime}$ is $U$-subinjective by the fact that the subinjectivity domain of each right module is closed under homomorphic images over hereditary rings (see [2, Theorem 2.1]). Since $R$ is right hereditary and $M^{\prime}$ is reduced, $\operatorname{Hom}\left(U, M^{\prime}\right)=0$, contradicting our assumption. Therefore, $M^{\prime}$ is indigent, and so $M$ is.

Case II. $\operatorname{Hom}(E, U) \neq 0$. Then $U$ is homomorphic image of an injective right $R$-module. By [8, Lemma 2.3], $U$ is F-subinjective for every projective module $F$. As $U$ is indigent, every projective right $R$-module is injective, i.e. $R$ is QF-ring. Then, $U$ is a unique singular simple right $R$-module. By [8, Theorem 3.1], there is a ring direct sum $R \cong S \times T$, where $S$ is semisimple Artinian ring and $T$ is an indecomposable matrix ring over a local QF-ring.

$(2) \Rightarrow(1)$ In case $R$ is a right V-ring, every simple right are module is injective, and we are done. For case 2-(ii), it is obvious. Assuming 2-(iii). Then $R$ has a 
unique non-injective simple right $R$-module, say $U$. $U$ is indigent by [1, Proposition $32]$.

In the preceding Theorem, $2-(i i)$ is equivalent to say that $R$ is right Hereditary righ Noetherian ring with a unique non-injective simple module (up to isomorphism) and every reduced right $R$-module $M$ with $\operatorname{Soc}(M) \neq 0$ is indigent. Furthermore, I would point out that a ring which has a non-injective simple indigent right module is right Noetherian. Note that over a commutative ring $R$ a simple module $S$ is pure-injective and $S \cong S^{+}$(see [5]). We have the following result by Theorem 18 and [9, Theorem 5.2].

Corollary 19. Let $R$ be a commutative ring. The following statements are equivalent.

(1) Every simple module is injective or indigent.

(2) $R$ is a $V$-ring, or $R \cong S \times T$, where $S$ is semisimple Artinian ring and $T$ is a DVR, or a local QF-ring.

Proposition 20. Let $R$ be a right nonsingular ring which is not right $V$-ring. The following are equivalent.

(1) Every simple module is indigent or injective.

(2) Every uniform module with nonzero socle is indigent or injective.

(3) Every indecomposable module with nonzero socle is indigent or injective.

Proof. In the general case there is the following chain of inclusions: simple modules $\varsubsetneqq$ uniform modules $\varsubsetneqq$ indecomposable modules. Thus, $(3) \Rightarrow(2)$ and $(2) \Rightarrow(1)$ are obvious. To $(1) \Rightarrow(3)$, let $M$ be an indecomposable non injective module with $\operatorname{Soc}(M) \neq 0$. Note that, by Theorem 18, $R$ is right hereditary and right Noetherian ring with unique non injective indigent simple module, say $U$. Then $M$ must be reduced. We will show that $M$ is indigent by mimicking second paragraph of the proof of (2-(ii)) in Theorem 18. Note that $\operatorname{Hom}(V, M)=0$ for each simple module $V$, because otherwise $V$ is a direct summand of $M$, this would contradict the fact $M$ is indecomposable. Therefore, $\operatorname{Hom}(U, M) \neq 0$, because otherwise $\operatorname{Soc}(M)=0$, contradicting its choice.

Assume that $M$ is not indigent. Then there is a non injective right $R$-module $K$ such that $M$ is $K$-subinjective. $\operatorname{Hom}(K, U) \neq 0$, otherwise $K$ is injective by indiginity of $U$. Then $M$ is $U$-subinjective by the fact that the subinjectivity domain of each right module is closed under homomorphic images over hereditary rings (see [2, Theorem 2.1]). Since $R$ is right hereditary and $M$ is reduced, $\operatorname{Hom}(U, M)$ must be zero, a contradiction. Thus, $M$ is indigent.

Proposition 21. A noninjective simple module over commutative ring is singular.

Proof. Let $U$ be a noninjective simple module. Recall that every simple module is either projective or singular. Assume that $U$ is a projective module. Then $U^{++}$is injective because $U \cong U^{+}$and the character module of a flat module is injective. $U$ is a pure submodule of $U^{++}$by [10, Proposition 5.3.9]. Then, $U$ is an injective module by the fact that pure injective absolutely pure modules are injective, a contradiction. Therefore, $U$ is singular.

Proposition 22. Let $R$ be a commutative nonsingular ring which is not $V$-ring. The following are equivalent. 
(1) Every simple module is indigent or injective.

(2) Every singular module is indigent or injective.

Proof. (1) $\Rightarrow(2)$ By Theorem [18, $R$ is hereditary Noetherian ring with unique noninjective indigent simple module, say $U$. By Proposition 21, $U$ is (unique) singular. Let $M$ be a noninjective singular module. Note that a module is indigent if it has an indigent direct summand. We may assume, without loss of generality, that $M$ is reduced. Since $R$ is hereditary Noetherian ring, $R$ is C-ring, i.e. every singular module has nonzero socle (see [16, Proposition 5.4]). Then $\operatorname{Hom}(U, M) \neq 0$. By mimicking second paragraph of the proof of Proposition 20, it is obtained that $M$ is indigent. (2) $\Rightarrow(1)$ follows by Proposition 21 .

In [1, Theorem 14], it is shown that $R$ is a QF-ring isomorphic to a matrix ring over a local ring if and only if $\mathrm{R}$ is right Artinian with homogeneous right socle containing $Z\left(R_{R}\right)$ essentially, and every simple right module is indigent or injective. We have the following result by Theorem 18 .

Corollary 23. Let $R$ be a ring which is not right $V$-ring. Assume that $Z\left(R_{R}\right) \neq 0$. The following are equivalent.

(1) Every simple right $R$-module is indigent or injective.

(2) $R \cong S \times T$, where $S$ is semisimple Artinian ring and $T$ is an indecomposable matrix ring over a local QF-ring.

(3) The left-hand version of (1).

\section{Acknowledgments}

This work was supported by Research Fund of the Çukurova University (Project Number:10871).

\section{REFERENCES}

[1] R. Alizade, E. Büyükaşik, and N. Er. Rings and modules characterized by opposites of injectivity. J. Algebra, 409:182-198, 2014.

[2] F. Altinay, E. Büyükaşik, and Y. Durğun. On the structure of modules defined by subinjectivity. J. Algebra Appl., (accepted), 2018.

[3] M. Auslander and M. Bridger. Stable module theory. No. 94. American Mathematical Society, Providence, R.I., 1969.

[4] P. Aydoğdu and S. R. López-Permouth. An alternative perspective on injectivity of modules. J. Algebra, 338:207-219, 2011.

[5] T. J. Cheatham and J. R. Smith. Regular and semisimple modules. Pacific J. Math., 65(2):315-323, 1976.

[6] T. J. Cheatham and D. R. Stone. Flat and projective character modules. Proc. Amer. Math. Soc., 81(2):175-177, 1981.

[7] P. M. Cohn. On the free product of associative rings. Math. Z., 71:380-398, 1959.

[8] Y. Durğun. Rings whose modules have maximal or minimal subprojectivity domain. J. Algebra Appl., 14(6):1550083, 12, 2015.

[9] Y. Durğun. An alternative perspective on flatness of modules. J. Algebra Appl., 15(8):1650145, $18,2016$.

[10] E. E. Enochs and O. M. G. Jenda. Relative homological algebra, volume 30. Walter de Gruyter \& Co., Berlin, 2000.

[11] C. Faith and D. V. Huynh. When self-injective rings are QF: a report on a problem. J. Algebra Appl., 1(1):75-105, 2002.

[12] C. Holston, S. R. López-Permouth, J. Mastromatteo, and J. E. Simental-Rodriguez. An alternative perspective on projectivity of modules. Glasgow Math. J., 57(1):83-99, 2015.

[13] D. V. Huynh. Structure of some Noetherian SI rings. J. Algebra, 254(2):362-374, 2002.

[14] S. R. López-Permouth, J. Mastromatteo, Y. Tolooei, and B. Ungor. Pure-injectivity from a different perspective. Glasg. Math. J., 60(1):135-151, 2018. 
[15] E. Matlis. Injective modules over Noetherian rings. Pacific J. Math., 8:511-528, 1958.

[16] J. C. McConnell and J. C. Robson. Noncommutative Noetherian rings, volume 30. American Mathematical Society, Providence, RI, 2001.

[17] C. Megibben. Absolutely pure modules. Proc. Amer. Math. Soc., 26:561-566, 1970.

[18] G. Puninski, M. Prest, and P. Rothmaler. Rings described by various purities. Comm. Algebra, 27(5):2127-2162, 1999.

[19] J. Rotman. An Introduction to Homological Algebra. Academic Press, New York, 1979.

[20] J. Simmons. Cyclic-purity: a generalization of purity for modules. Houston J. Math., 13(1):135-150, 1987

[21] E. G. Skljarenko. Relative homological algebra in the category of modules. Uspehi Mat. Nauk, 33(3(201)):85-120, 1978.

[22] B Stenström. Coherent rings and fp-injective modules. J. London Math. Soc. (2), 2:323-329, 1970.

[23] J. Trlifaj. Whitehead test modules. Trans. Amer. Math. Soc., 348(4):1521-1554, 1996.

[24] R. B. Warfield, Jr. Purity and algebraic compactness for modules. Pacific J. Math., 28:699719, 1969.

Çukurova University, Department of Mathematics, 01330, Adana, Turkey.

E-mail address: ydurgun@cu.edu.tr 